\title{
Filoviruses are ancient and integrated into mammalian genomes
}

\author{
Derek J Taylor ${ }^{* 1}$, Robert W Leach² and Jeremy Bruenn
}

\begin{abstract}
Background: Hemorrhagic diseases from Ebolavirus and Marburgvirus (Filoviridae) infections can be dangerous to humans because of high fatality rates and a lack of effective treatments or vaccine. Although there is evidence that wild mammals are infected by filoviruses, the biology of host-filovirus systems is notoriously poorly understood. Specifically, identifying potential reservoir species with the expected long-term coevolutionary history of filovirus infections has been intractable. Integrated elements of filoviruses could indicate a coevolutionary history with a mammalian reservoir, but integration of nonretroviral RNA viruses is thought to be nonexistent or rare for mammalian viruses (such as filoviruses) that lack reverse transcriptase and replication inside the nucleus. Here, we provide direct evidence of integrated filovirus-like elements in mammalian genomes by sequencing across host-virus gene boundaries and carrying out phylogenetic analyses. Further we test for an association between candidate reservoir status and the integration of filoviral elements and assess the previous age estimate for filoviruses of less than 10,000 years.

Results: Phylogenetic and sequencing evidence from gene boundaries was consistent with integration of filoviruses in mammalian genomes. We detected integrated filovirus-like elements in the genomes of bats, rodents, shrews, tenrecs and marsupials. Moreover, some filovirus-like elements were transcribed and the detected mammalian elements were homologous to a fragment of the filovirus genome whose expression is known to interfere with the assembly of Ebolavirus. The phylogenetic evidence strongly indicated that the direction of transfer was from virus to mammal. Eutherians other than bats, rodents, and insectivores (i.e., the candidate reservoir taxa for filoviruses) were significantly underrepresented in the taxa with detected integrated filovirus-like elements. The existence of orthologous filoviruslike elements shared among mammalian genera whose divergence dates have been estimated suggests that filoviruses are at least tens of millions of years old.

Conclusions: Our findings indicate that filovirus infections have been recorded as paleoviral elements in the genomes of small mammals despite extranuclear replication and a requirement for cooption of reverse transcriptase. Our results show that the mammal-filovirus association is ancient and has resulted in candidates for functional gene products (RNA or protein).
\end{abstract}

\section{Background}

The ongoing threat of emerging hemorrhagic diseases has made the search for reservoir species with a history of coevolution with filoviruses a priority [1,2]. Outbreaks of filovirus infections are known from Africa and the Phillipines [3-5] and, in some cases, the mortality of primates is so severe as to raise concerns of extinction [5]. Bats are considered a candidate for a reservoir based on the detection of filovirus-specific RNA, antibodies, and viral parti-

* Correspondence: djtaylor@buffalo.edu

1 Department of Biological Sciences, The State University of New York at Buffalo, Buffalo, NY 14260, USA

Full list of author information is available at the end of the article cles $[1,6-10]$. Still, the average seroprevalence in tested bats is much smaller than expected (usually $<5 \%$ ) for large colonies of a main reservoir [6], and the ability of bats to maintain a persistent hypovirulent infection is unknown. Rodents and insectivores (shrews) have further been proposed as the leading candidates for filovirus reservoirs by modeling, the detection of filovirus RNA, and in one specimen, the potential detection of a DNA copy $[2,11]$. Rodents (mice and guinea pigs) share one expected feature of coevolution -- asymptomatic infections from wild-type filoviruses [12]. However, a reservoir role for rodents and shrews has been questioned because only one study has detected filovirus RNA frag- 
ments in these small mammals, and many more outbreaks than observed are expected from a rodent reservoir that is commensal with humans [7]. Moreover, no live viruses, filovirus particles or antibodies to filoviruses have been found in rodents or shrews. Distinguishing principal reservoir species from "spillover" infections remains a challenge.

Filoviruses are a family of non-segmented negative sense RNA viruses with filamentous virions (Fig. 1). The protein-coding genes in the filovirus genomes (3'-NP, VP35, VP40, GP, VP30, VP24, and L protein-5') have a transcriptional gradient from NP to L protein [13]. The two major evolutionary groups of filoviruses have been assigned to the genera Ebolavirus and Marburgvirus. Filoviruses are estimated to have diverged for less than 10,000 YA [14]--about the same timescale as the rise of agriculture. Although high mutation rates in RNA viruses have shrouded nearly every interfamilial relationship, the Order Mononegavirales, which contains Filoviridae, is an exception [13]. Here, filoviruses show significant sequence similarity to some of the Paramyxoviridae such as Morbillivirus (e.g. measles and Rinderpest viruses) [15]. Notably, the N-terminal 450 amino acid residues of NP, which is examined in the present study, shows significant conservation among the Mononegavirales and is needed for self-assembly of the nucleoprotein [16].

There are now several cases in eukaryotes where nonretroviral integrated RNA viruses (NIRVs) have been detected $[17,18]$. Still, this type of transfer is believed to be extremely rare in mammals $[17,19]$ because the process requires the cooption of reverse transcriptase and perhaps replication within the nucleus. The sole mammalian example is bornavirus, which is unique among RNA viruses of animals in developing persistent infections within the nucleus. The study of NIRVs requires an evolutionary approach where the direction of transfer is tested. Evolutionary comparisons among NIRVs have been carried out for the Totiviridae in yeast [20], and the Bornaviridae in mammals [17]. In the Totivirus system there strong support for the direction of transfer from virus to fungus, and a role for the expression of NIRVs in viral interference has been proposed [20]. We proposed that NIRVs are more common than presently known and might be detected in other systems with persistent infections of non-retroviral RNA viruses. As part of a search for NIRVs in NCBI databases we found strong BLAST matches of NP sequences from filoviruses to translated genomic sequences from small mammals. We aimed to test if these sequence similarities might indicate NIRVs of filoviruses.

\section{Results and Discussion}

tBLASTn with Marburgvirus NP amino acid sequence yielded matches with low expect values (as low as 10-49), indicating that similarity is unlikely to be a chance result. We found twenty matches with expect values less than the standard "significance" value of $10^{-5}$ (see Fig. 2). The tammar wallaby, (Macropus eugenii) showed the strongest similarity (49.4\% identity) and also had at least 12 different strong sequence matches. The little brown bat (Myotis lucifugus) had four significant matches, while the guinea pig (Cavia porcellus), Ord's kangaroo rat (Dipodmys ordii), the common shrew (Sorex araneus), and the gray short-tailed opossum (Monodelphis domestica; Chromosome 2) each had single matching sequences with expect values $<10^{-5}$. Another marsupial, the common brushtail possum (Trichosurus vulpecula) had six strong matches from the Expressed Sequence Tags (EST) database. All but three of these sequences (including the EST matches) had at least one apparent disruption of the open reading frame (ORF). tBLASTn with the L protein yielded one value with a low expect value $\left(10^{-74}\right)$, the gray short-tailed opossum (Monodelphis domestica; Chromosome 3). A tBLASTn search using the best matching placental mammal match from the original NP search as a query sequence also yielded strong matches in mammals: the pygmy hedgehog tenrec (Echinops telfairi), the mouse (Mus musculus) and the brown rat (Rattus norvegicus).

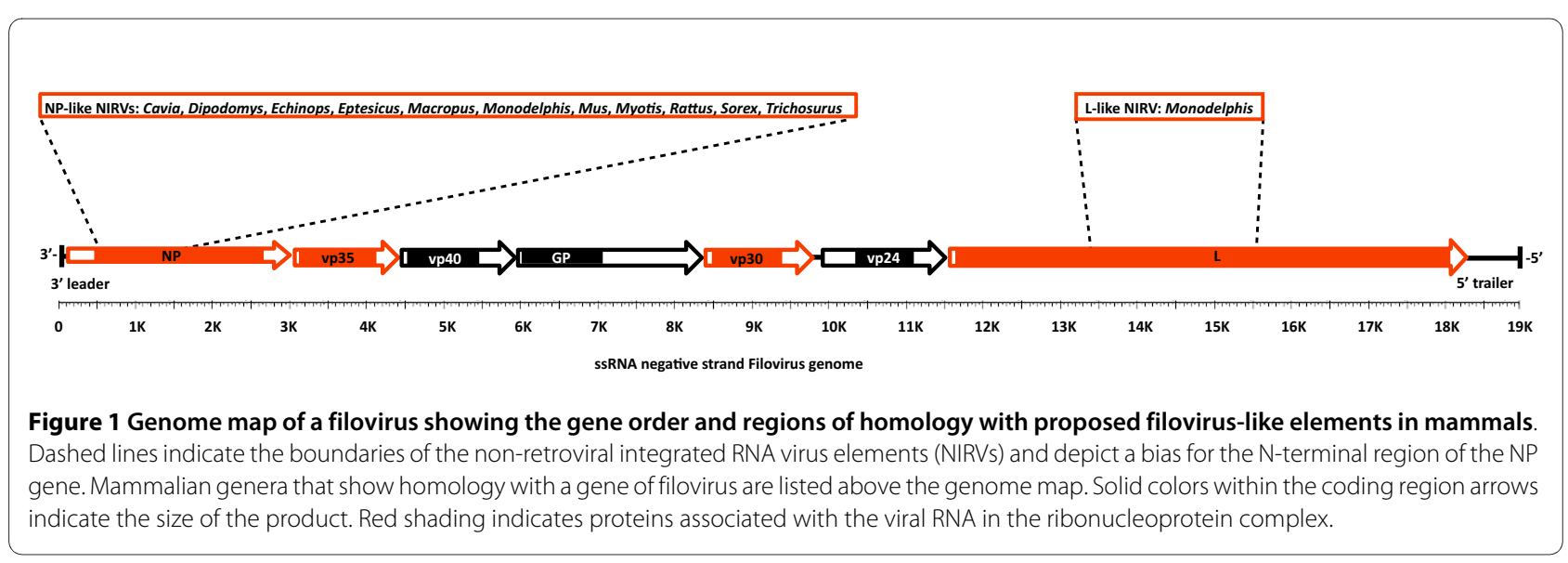




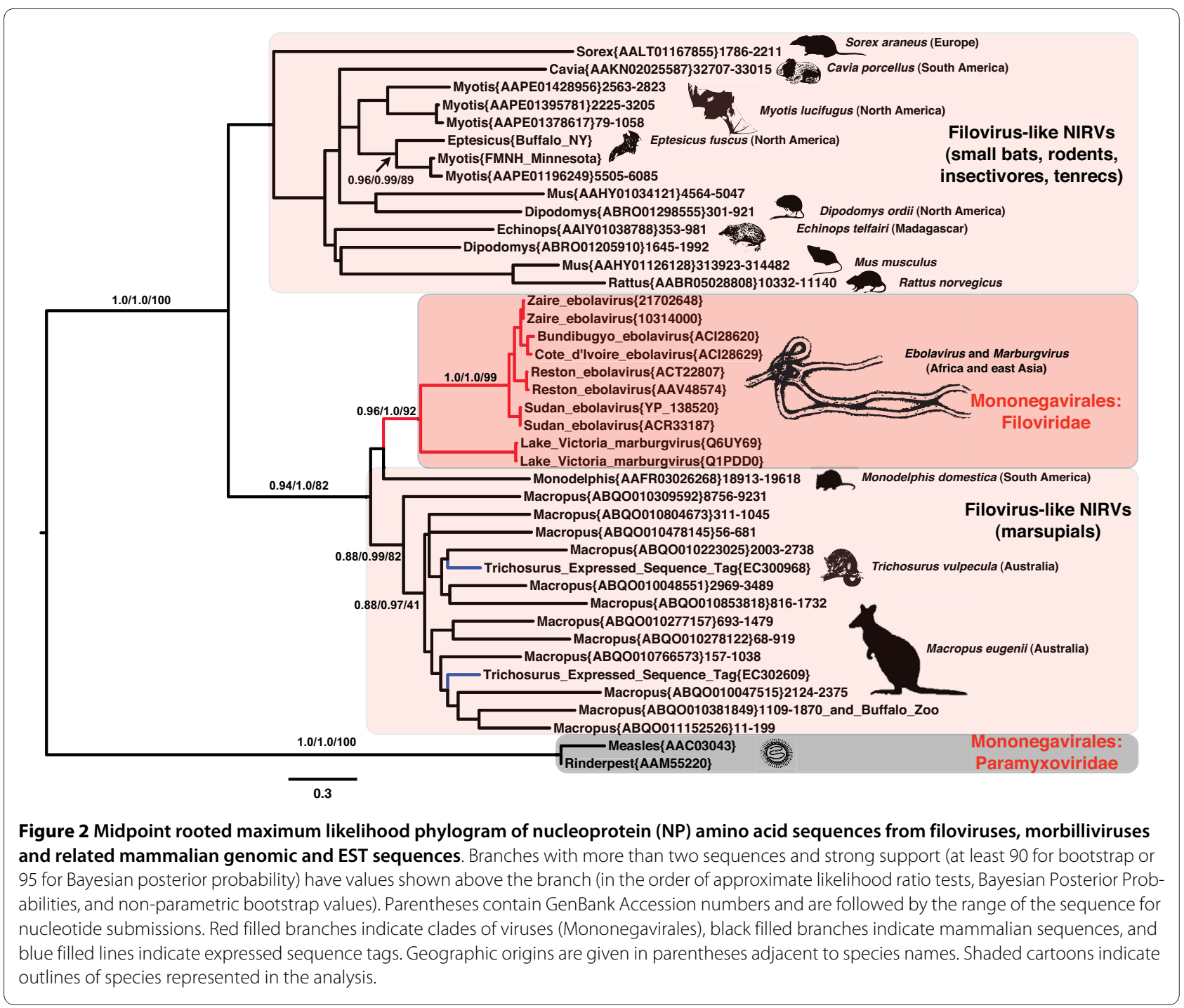

The filovirus-like EST nucleotide sequences from the common brushtail possum had a BLAST match to a single region of the wallaby genome with longest match (DY609334) having a 78\% identity (9\% of mismatches are gaps) for 662 bases.

We tested for integrated DNA based copies of the filovirus-like sequences in the two mammals with the most copies, the tammar wallaby and the little brown bat. We designed PCR primers from mammalian genomic sequence flanking the longer BLAST matches and carried out PCR amplification of DNA extractions from different specimens than used for existing genome projects. Our sequence of the tammar wallaby had only a single transition difference from the genome project sequence. The sequence of the little brown bat from Minnesota (FMNH 172384) had a similarity of $96 \%$ with four indels compared to contig (AAPE01196249) from the existing genome. To test for the presence of a filovirus-like DNA sequence in an additional insectivorous bat, we extracted
DNA from a specimen of big brown bat (Eptesicus fuscus). Using primers designed from the little brown bat, we again obtained PCR product and sequence. In this case, the identity between the sequences of the two genera of bats was $87 \%$ with 11 indels. In each case the similarity of the new sequences obtained from DNA to genomic sequence is consistent with an integrated filovirus-like DNA copy in these mammalian genomes.

We next carried out a phylogenetic analysis of the NP and $\mathrm{L}$ protein amino acid sequence alignments with Mononegavirales (paramyxovirids and filovirids) to assess the direction of the transfer. Because the $\mathrm{L}$ protein gene is known to be the most conserved gene in the Mononegavirales, a large number of BLAST matches with expect values $<10^{-5}$ was found between the families of Mononegavirales in L protein compared to the NP. The midpoint rooted maximum likelihood (ML) phylogram placed the potential mammalian NIRVs within the Mononegavirales, and revealed that the mammalian 
sequences are more closely related to filoviruses than to Paramyxoviruses (Figs. 2, 3). Indeed the L protein-like sequence from Monodelphis was more closely related on the best ML tree to Marburgvirus than to other known filoviruses (i.e., Ebolavirus) (Fig. 3). This result suggests that the most recent integration of filoviruses from our data involves South American marsupials. The NP analysis also revealed that the South American Monodelphis is more closely related to known filoviruses than to other mammalian sequences (Fig. 3 and Additional file 1: Fig. S1). Although many of the sequences are of different lengths in the NP alignment (Additional file 2: Fig. S2), it is now well known that sequences of very different lengths can be accurately placed on phylogenies [21]. However, there could be long-branch effects or alignment effects for the NP phylogeny as the exclusion of the distantly related Morbillivirus sequences yielded the same mammalian paraphyly, but increased the support values (Fig. 4). For both genes, the placement of the mammalian NIRVs with the filoviruses (i.e. within Mononegavirales) had maximum support for each measure of reliability. The placement and the strong support values for this node are consistent with the direction of transfer from viruses (Mononegavirales) to mammalian genomes. Endogenous reverse transcriptase activity has been shown experimentally to integrate non-retroviral RNA viruses in mammals $[17,22]$ and may have played a role in filovirus integration. Interestingly, the closest flanking coding regions of integrated filovirus-like elements to at least five of the NIRV's of Macropus, and the separate NP and L-like NIRVs of Monodelphis, are truncated or disrupted non-LTR retrotransposons of the LINE-1 family. Our results represent the first case of NIRV formation in mammals with a virus that has extranuclear replication [17].

The observation that most of the mammalian sequences have ORF disruptions and possess only truncated NP-like genes (Fig. 1) is also inconsistent with a transfer from mammals to virus. Only Monodelphis has more than one different filovirus-like gene (Additional file 3: Fig. S3) and these (the NP and L protein-like sequences) are on separate chromosomes. The apparent genic bias of NIRVs for the NP gene could have a biological explanation. Because of the transcription gradient in the Mononegavirales, the most common primary transcript is NP [13]. We also note that experimental expression of an N-terminal portion of the Ebolavirus NP gene (from residue 1-450 in wildtype NP) that is positionally homologous to the region of NP spanned by mammalian NIRVs (from residue 18-405 in wildtype NP, NP_066243) is sufficient to inhibit the formation of Ebolavirus minigenomes in a dosage specific fashion [23]. A background

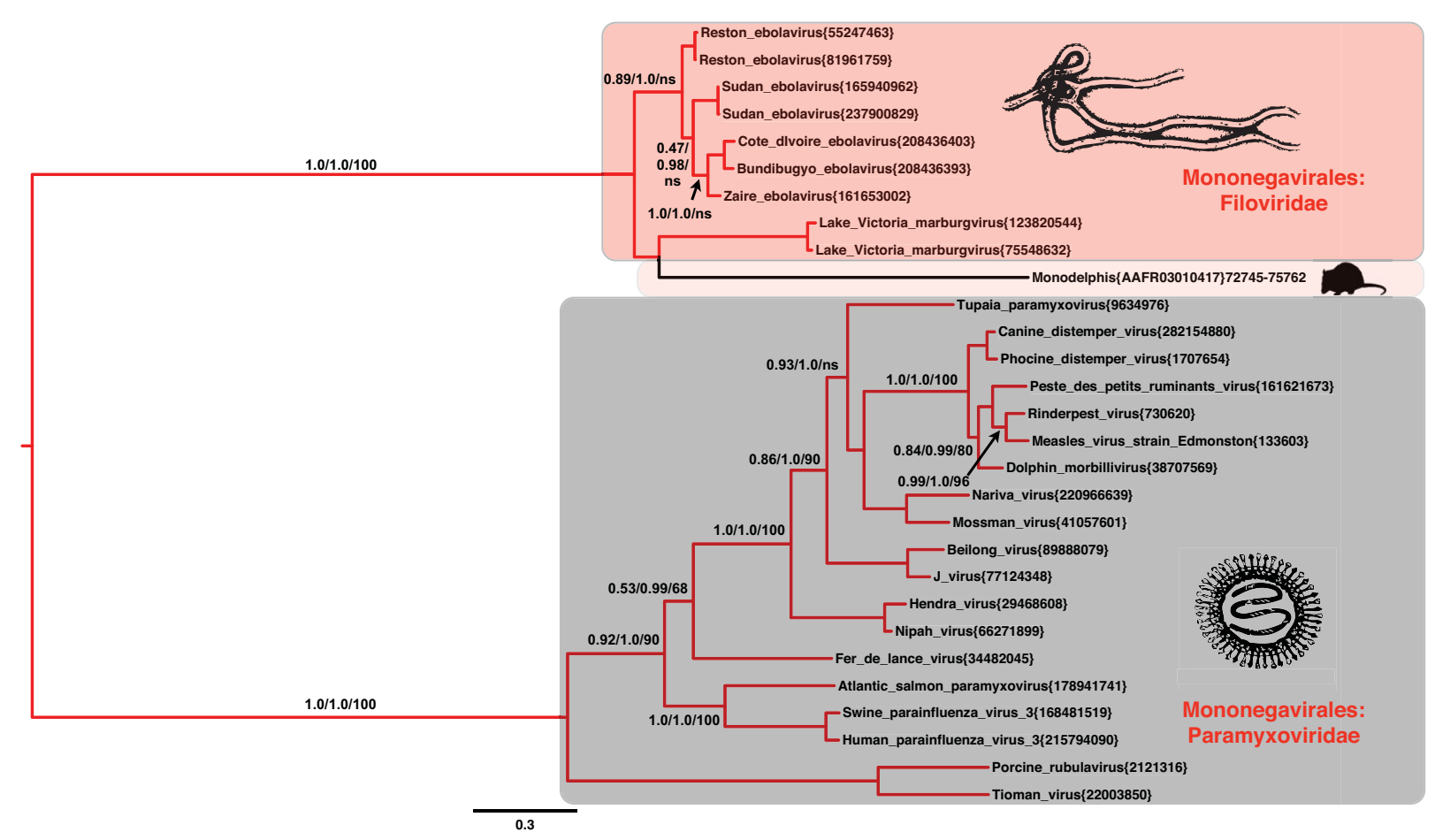

Figure 3 Midpoint rooted maximum likelihood phylogram of $L$ protein amino acid sequences from filoviruses, Paramyxoviridae, and a South American marsupial genomic sequence. Labeling and shading details are as in Fig. 2 except that the species name and continent for the mammalian sequence are provided in the caption: Monodelphis domestica (South America). 


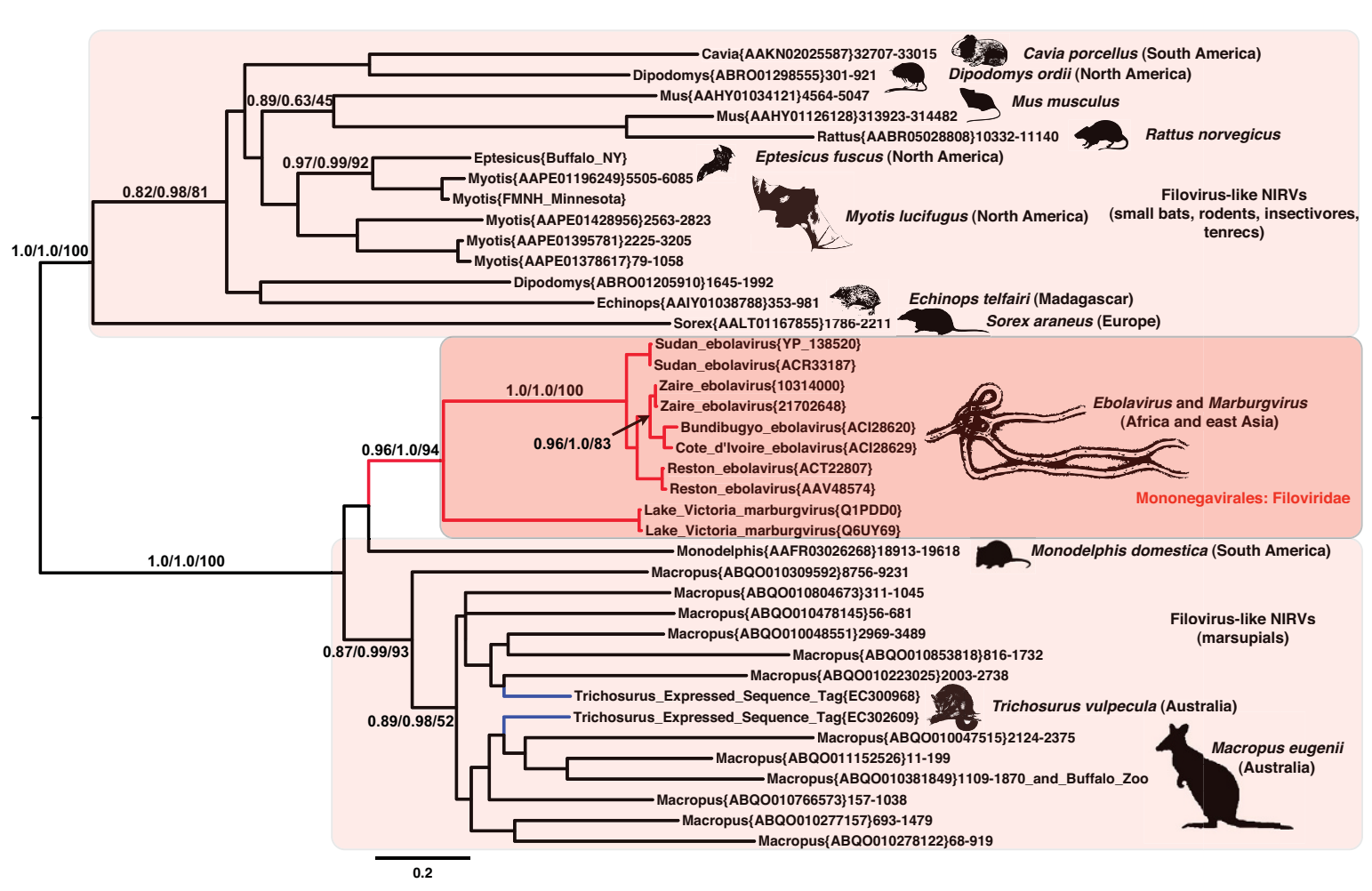

Figure 4 Midpoint rooted maximum likelihood phylogram of nucleoprotein (NP) amino acid sequences from filoviruses and related mammalian genomic and EST sequences showing the paraphyly of mammals. Branches with more than two sequences and strong support (at least 90 for bootstrap or 95 for Bayesian posterior probability) have values shown above the branch (in the order of approximate likelihood ratio tests, Bayesian Posterior Probabilities, and non-parametric bootstrap values). Parentheses contain GenBank Accession numbers and are followed by the range of the sequence for nucleotide submissions. Red filled branches indicate clades of viruses (Mononegavirales), black filled branches indicate mammalian sequences, and blue filled lines indicate expressed sequence tags. Geographic origins are given in parentheses adjacent to species names. Shaded cartoons indicate outlines of species represented in the analysis.

transcription bias could account for overrepresentation in NIRVs of NP, but such a bias fails to explain the N-terminal bias within the NIRVs of NP. The bias is consistent with the experimental filoviral interference mechanism involving the N-terminal of NP.

Despite ORF disruptions, it is clear that at least some mammalian filovirus-like NIRVs of NP are expressed. In the marsupial Trichosurus, we detected six different NPlike ESTs (EC302609, DY609334, EC300968, EC310159, DY613238, EC352436) from three tissue-specific cDNA libraries: liver, spleen/lymphatic system and gonads. These tissues play an important role in the pathology and replication of filoviruses [24]. We did not detect the NIRV in the cDNA libraries made from brain, whole embryo, kidney, uterus/reproductive tract, or gut tissues. Still, non-functional pseudogenes can be transcribed by interactions with neighboring functioning loci [25]. We tested for selective maintenance of codon structure in the filovirus-like NIRVs as a further indication of function. Comparisons of rates of amino-acid changing substitutions $\left(\mathrm{d}_{\mathrm{N}}\right.$ or $\left.\mathrm{K}_{\mathrm{a}}\right)$ to rates of silent substitutions $\left(\mathrm{d}_{\mathrm{S}}\right.$ or $\left.\mathrm{K}_{\mathrm{s}}\right)$ do bear the signature of selective codon maintenance or purifying selection. Non-functional regions should conform to neutral expectations where $d_{N}=d_{S}$ and $d_{N} / d_{S}=1$ [26]. For regions undergoing purifying selection, the silent substitution rate should prevail whereby $d_{N}-d_{S}<<0$ and $d_{N} / d_{S}<<1$. The codon-based test of neutrality using the model of Kumar (which accommodates transition/ transversion rate bias) indicates that silent mutations are significantly overrepresented in an alignment of filoviruslike NIRVs $\left(\mathrm{d}_{\mathrm{N}}-\mathrm{d}_{\mathrm{S}}=-9.427, \mathrm{P}<0.001\right)$ [27]. Likewise, Bayesian calculations of site-specific $\mathrm{K}_{\mathrm{a}} / \mathrm{K}_{\mathrm{s}}$ using evolutionary models that accommodate codon usage differences [28], reveal a prevailing pattern of values significantly less than 1 (Fig. 5). Under a model that allows purifying, neutral and positive selection (Model M8), the distribution of $\mathrm{K}_{\mathrm{a}} / \mathrm{K}_{\mathrm{s}}$ peaks at about 0.4. For the M8 model, 67 percent of these alignment sites (and all of the M7 sites) have upper 95 percent confidence limits for $<1$. While these $\mathrm{K}_{\mathrm{a}} / \mathrm{K}_{\mathrm{s}}$ values are larger than is typical of strong purifying selection, they are markedly less than 


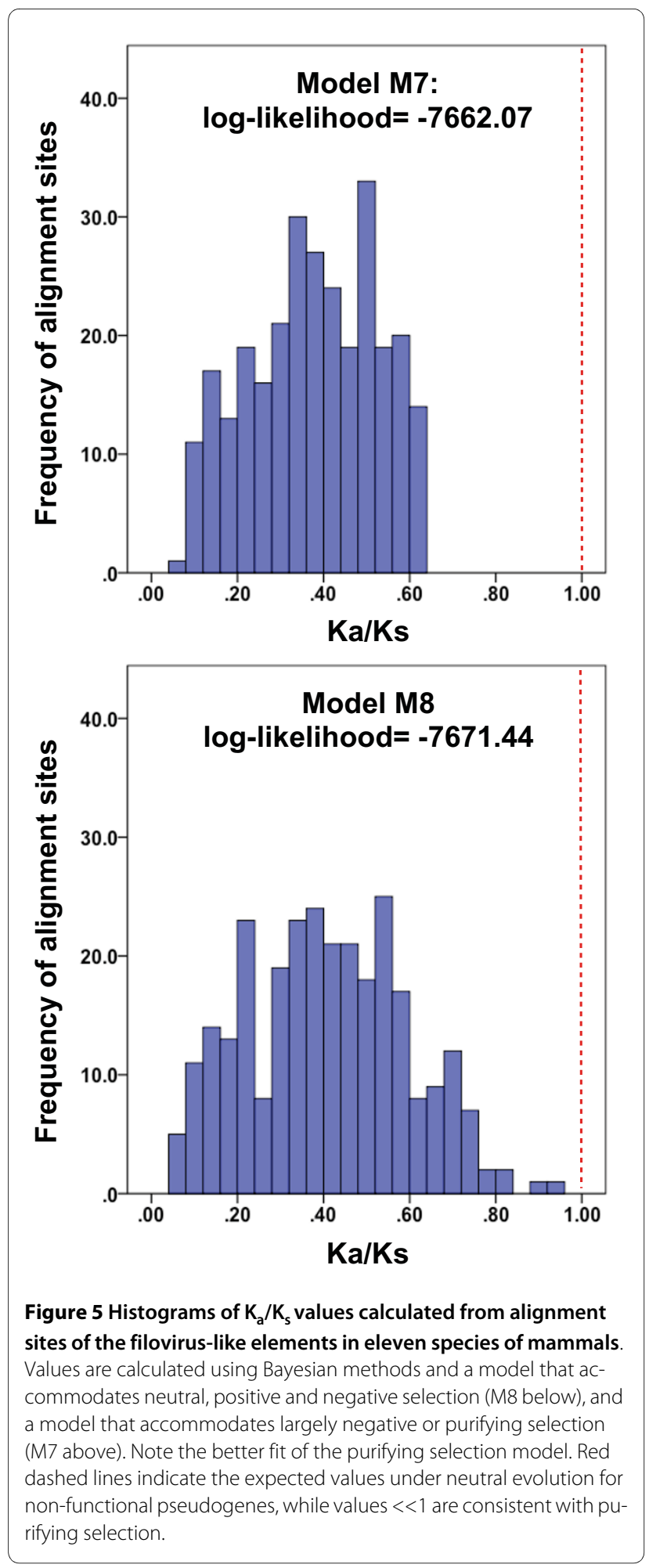

neutral expectations or even the range of $\mathrm{K}_{\mathrm{a}} / \mathrm{K}_{\mathrm{s}}=0.6$ to 1.0 that is reported for disrupted transcribed pseudogenes in mammals [29]. Even though there appears to be selection for preserving codons, the tests cannot differentiate between past and present function. Moreover, the products need not be protein-based -- RNA interference products can elicit codon-like selection to interact with protein-coding genes [29]. The functionality and potential role of NIRVs in the well-known resistance to filoviruses of some NIRV-containing mammals (mice and guinea pigs) will have to be addressed with experiments.

More than one endogenization is required to account for the paraphyly of mammals and the paraphyly of marsupials with filoviruses. The finding of a monophyletic clade for placental mammals with samples from several continents requires a single ancient integration with several losses of NIRV signal or multiple integrations of a related virus in unrelated mammal groups (Fig. 6). A single origin for eutherian NIRVs is supported by the rarity of the process -- endogenization of non-retroviral RNA viruses with extranuclear replication is previously unknown in mammals. Ancient transcribed pseudogenes $>100$ million years old are known from mammals [29] and the primate bornavirus integration is believed to be older than 40 million years [17]. Although much of the deeper groupings have weak support and there has been gene duplication, there are some well-supported groupings that agree with mammalian phylogeny. The strongly supported groups are the two bat genera, the genera of mouse-like rodents, and the Australian marsupials, Trichosurus and Macropus. These genera of marsupials are believed to have shared a common ancestor from 39 to 52 million years ago [30]. A clear indicator of antiquity is the syntenous genomic location of a rat and mouse filovirus-like NIRV (Fig. 7A, B). These are the same copies that have a sister group relationship (Fig. 2). It is unlikely that integration of filovirus NP genes at the same genomic position occurred independently in rats and mice. The rat-mouse orthology provides a minimum date of NIRV formation at 12 to $24 \mathrm{MY}[31,32]$. Of the species with filovirus-like elements only the rat, mouse and Monodelphis have detailed chromosomal maps, but further mapping and taxonomic sampling will permit a more robust assessment of the age of eutherian NIRVs. Still, we conclude that the association between filoviruses and mammals is likely to be 10's of millions of years older than the previous estimate. Filoviruses join bornaviruses as the only demonstrated prehistoric non-retroviral RNA viruses.

The eutherian orders with NIRVs of filoviruses closely match the proposed candidate reservoir groups of bats, rodents, and insectivores [1,2] (Fig. 6). This pattern is not a sampling artifact that we can attribute to the available genome assemblies. Seven of the ten genomes (including the Big Brown bat) sampled from predicted reservoir orders had integrated filoviruses, while only 1 of 27 from non-candidate eutherian orders had detected integrated filovirus-like elements (Fisher's exact test, two-tailed $\mathrm{p}$ value $=0.00003$ ). The sole eutherian species from a non- 


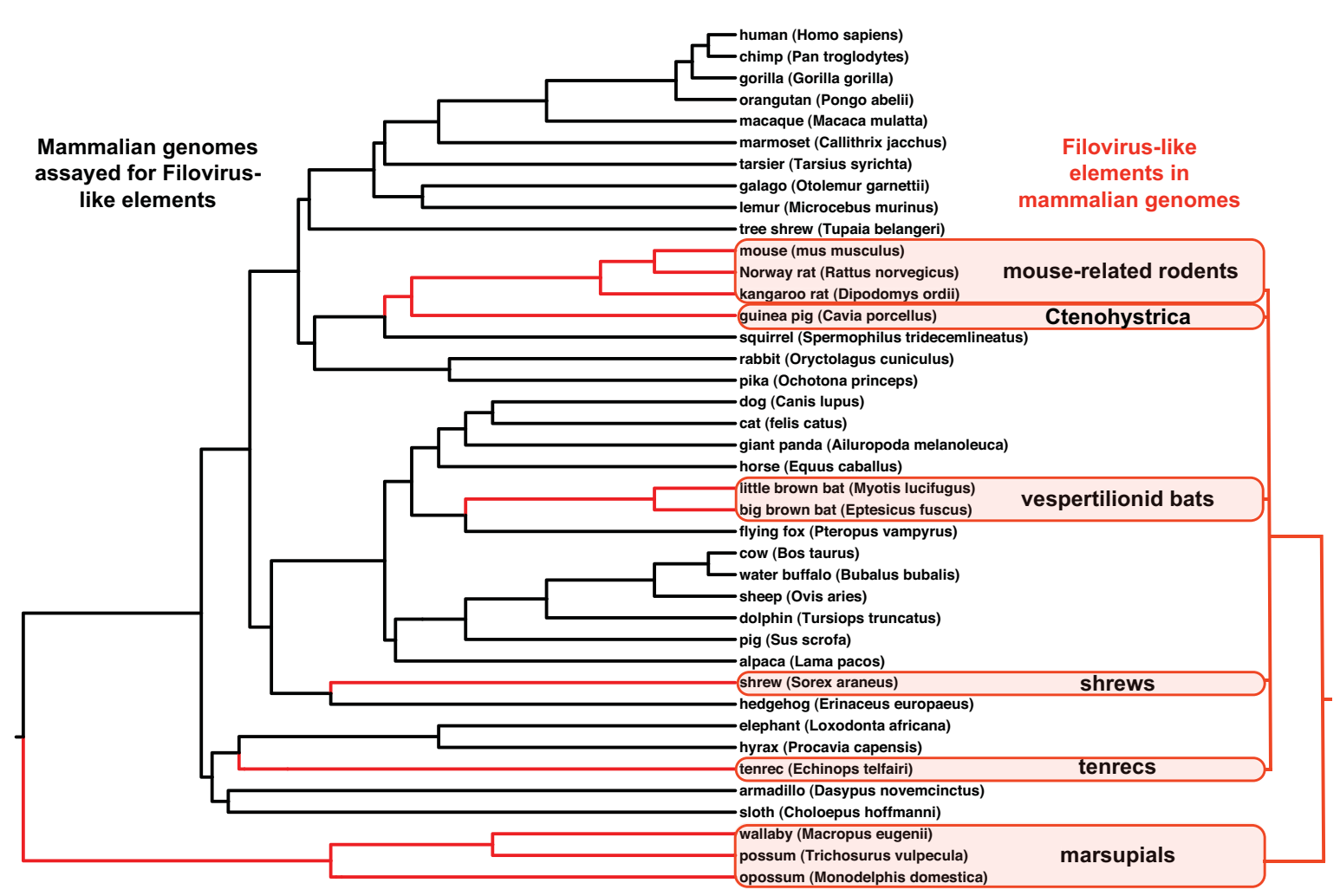

Figure 6 Summary graph showing mammalian genomes assayed for filovirus-like elements and the phylogenetic distribution of the mammals with filovirus-like elements. Red shading indicates that species with detected filovirus-like elements fall into a marsupial and a eutherian group. The platypus genome was also assayed but is not depicted here. The mammalian phylogeny is based on a composite of recent studies $[30,44,45]$

candidate group to have a potential NIRV was the pygmy hedgehog tenrec, which is the Afrotherian small insectivore analog on the island of Madagascar. The three assemblies of genomes from candidate orders that lacked apparent NIRVs were the ground squirrel (Spermophilus tridecemlineatus), the European hedgehog (Erinaceus europaeus) and the fruit bat (Pteropus vampyrus). At present it is unclear why some small mammal groups (bats, rodents, insectivores and marsupials) appear to have an association with filoviruses. Still, the study of filovirus-like NIRVs could have predictive value for identifying filovirus reservoirs, ancestral proteins, outbreak modeling, undetected lineages of filoviruses and virulence in mammalian species. For example, the close relationship of South American and expressed Australian marsupial filovirus-like NIRVs with rapidly evolving African filoviruses now makes it more likely that the New World harbors undetected filoviruses or has acted as a source region for extant filoviruses.

\section{Conclusions}

Our findings indicate that filovirus infections are recorded as paleoviral elements in the genomes of small mammals. These elements are candidates for functional gene products (RNA or protein). The integration is unex- pected because filoviruses lack reverse transcriptase and the ability to replicate within the nucleus. Our results indicate that the association of mammals with filoviruses is likely tens of millions of years older than previously thought.

\section{Methods}

\section{Nucleic Acid Extractions}

DNA was extracted from freshly collected wallaby fur, toe clips of a Big Brown Bat, and DMSO preserved tissue from a little brown bat using the DNA Quickextract kit (Epicentre Technologies) modified to have a two hour incubation step at $65^{\circ} \mathrm{C}$.

\section{PCR, RTPCR, and DNA Sequencing}

$50 \mu \mathrm{l}$ PCR reactions contained $5 \mu \mathrm{L}$ of extracted DNA template, $25 \mu \mathrm{L}$ of $2 \times$ GoTaq PCR reagent mix (Promega) each primer. Primers for sequencing and PCR were: $5^{\prime}$ GCCTTGTCGACGTTCATCCTGTG-3' and 5'-GAGC CATTGGTTGCTCGGAAGC3- for Myotis; 5'-GGAGACCTCGAGCAAATGGAGC-3' and 5'-GAGCCATTGGTTGCTCGGAAGC-3' for Eptesicus and 5'-TGA GTTTTGGGGTGAATTAGC-3' and 5'-GGGTGACA TAGGGAAGCACA-3' for Macropus. The PCR temperature profiles were: 30 cycles of $94^{\circ} \mathrm{C}$ for $30 \mathrm{~s}, 50^{\circ} \mathrm{C}$ for $30 \mathrm{~s}$ 
A.

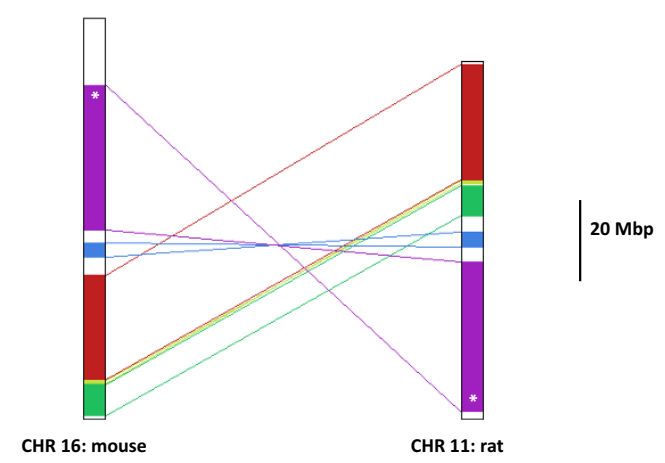

B.

$\underset{\text { Dgcr6 }}{\text { Prodh }}$

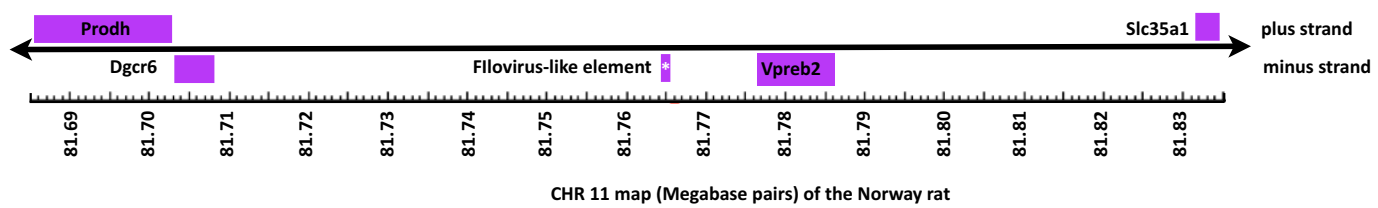

Figure 7 Chromosome maps showing synteny of regions flanking filovirus-like elements in rat and mouse genomes with a whole chromosome view (A) and a local view (B). White asterisks represent the locations of the phylogenetic sister copies of filovirus-like elements. Five synteny blocks with a reversal distance of 2 were found between CHR 16 of the mouse and CHR 11 of the rat. The filovirus-like elements are located on a reversed synteny block (purple shading). A close up view shows the flanking gene locations and acronyms.

and $72^{\circ} \mathrm{C}$ for $2 \mathrm{~min}$, and final extension at $72^{\circ} \mathrm{C}$ for $5 \mathrm{~min}$. PCR products were purified and sequenced by the University of Washington High Throughput Genomics Facility. Geneious 4.8 was used to assemble and edit electrophoregrams. New sequences from this study have been named as endogenous filovirus-like NP elements (EFLNP) and assigned the following Genbank accession numbers: HM545133-HM545135.

\section{Bioinformatics}

Initial searches for sequence similarity to filoviruses used protein sequences from genes of Marburgvirus (NC_001608.3) as a query with tBLASTn in the WGS database and the EST database and BLASTp in protein database of NCBI. A second tBLASTn in the same databases used the best scoring non-viral sequence of placental mammals as a query. A third search used the EST nucleotide sequences Trichosurus as a query for the nucleotide and WGS databases. Nonviral subject sequences with expect values of $\mathrm{E}<10^{-5}$ and two different sequences from each of the five known species in the
Filoviridae were retained for alignment. A search constrained to Mononegavirales NCBI Genomic Reference Sequences Marburgvirus (NC_001608.3) found two species of Morbillivirus had expect values below $10^{-5}$ (Rinderpest virus, and Measles virus) that were retained for alignment. L protein sequences searches used a similar strategy but many more Paramyxoviruses had a significant match to Marburgvirus. We retained 19 different Paramyxoviruses for alignment with filovirus and the mammal sequence using BLAST explorer [33].

For genome assembly sequences, the sequence boundaries and translations identified by tBLASTn were used to retrieve nucleotide sequences and assemble amino acid sequences. MAFFT [34] was used to align the protein sequences for all analyses using the default parameters. The NP alignment was trimmed to the range of the mammalian filovirus-like sequences and the L protein alignment which had a mosaic of conserved and length variable regions was trimmed by Gblocks [35] (with gaps allowed). 
Phylogenetic estimates were obtained with a maximum likelihood optimality criterion (PhyML [36] and RAxML [37]) and Bayesian MCMC methods [38]. Models were chosen according to the best available optimal model from Prottest [39] (ML) or using a mixed model prior for amino acids (Mr.Bayes). Reliability was assessed by nonparametric bootstrapping (ML), approximate likelihood ratio tests (aLRT: $\mathrm{SH}$ like tests), and posterior probabilities. Prottest determined that the $\mathrm{LG}+\mathrm{G}+\mathrm{F}$ model was the best fit with the AIC criterion for the L protein alignment and the JTT+G model was the best fit for the NP alignment. We therefore carried out maximum likelihood analysis using these models. However, as RAxML does not accommodate the LG model we used the next best fit model of RtREV $+\mathrm{G}+\mathrm{F}$ for the RAxML of $\mathrm{L}$ protein [40]. For bootstrapping, RAxML estimated the number of pseudoreplicates. For PhyML, both SPR and NNI search algorithms were used with five random starting trees. For Bayesian analysis, a million Markov chain Monte Carlo generations were initially carried out and convergence metrics were assessed. If the average standard deviation of split frequencies $<0.01$ and a plot of log-likelihood scores versus generation time as consistent with convergence, then we culled the burn-in set of half of the trees and calculated the posterior probabilities. We added $500,000 \mathrm{MCMC}$ generations at a time until convergence metrics were satisfied.

Tests of neutral evolution were carried out using both approximate methods (Codon-based Z test with Kumar model [27] that accommodates transition-transversion ratio bias) and Bayesian methods [28] of estimating sitespecific $\mathrm{K}_{\mathrm{a}} / \mathrm{K}_{\mathrm{s}}$. For input, codon alignments were estimated using PAL2NAL [41] from a subset of sequences from the amino acid sequence alignment. We used only one sequence per species in the alignment. As both MEGA and Selecton require continuous ORF's, disrupted codons were replaced with gaps. For the Bayesian estimate of $\mathrm{K}_{\mathrm{a}} / \mathrm{K}_{\mathrm{s}}$, an ML tree was input after estimating with PhyML and a GTR $+G$ model. Site-specific $K_{a} / K_{s}$ values were culled from the Macropus sequence sites, which reduced the influence of alignment end gaps on the estimates. A histogram of the $K_{a} / K_{s}$ values was created in PASW statistics 18.

To evaluate orthology between rat and mouse NIRVs, we used genomic BLAST searches and visualized the matches and annotations on the NCBI chromosome maps. Whole chromosome comparisons of larger orthologous blocks were assessed using the Cinteny server [42] and Roundup database [43].

\section{Additional material}

Additional file 1 Fig. S1. Maximum likelihood phylogram of nucleoprotein (NP) amino acid sequences from filoviruses and marsupial sequences.
Additional file 2 Fig. S2. Alignment of nucleoprotein (NP) amino acid sequences from filoviruses and related mammalian genomic and EST sequences. Disruptions to the open reading frame are shown by an "X". Additional file $\mathbf{3}$ Fig. S3. Alignment of $L$ protein amino acid sequences (culled in Gblocks) from filoviruses and related mammalian genomic sequence.

\section{Authors' contributions}

DJT and JB conceived the study, carried out the bioinformatics analysis, participated in lab experiments and co-wrote the paper. RWL designed software and carried out BLAST searches. All authors read and approved the manuscript.

\section{Acknowledgements}

We thank Gerald Aquilina and Kurt Volle at the Buffalo Zoo for fur samples from Macropus eugenii, Katharina Dittmar (University at Buffalo) for tissue samples from Eptesicus fuscus, the Field Museum of Natural History for tissue from Myotis lucifugus, and the administration team of the Center for Computational Research (University at Buffalo) for set up, monitoring, and use of the U2 cluster.

\section{Author Details}

'Department of Biological Sciences, The State University of New York at Buffalo, Buffalo, NY 14260, USA and 2 Center for Computational Research, The State University of New York at Buffalo, Buffalo, NY 14203, USA

Received: 4 June 2010 Accepted: 22 June 2010

Published: 22 June 2010

\section{References}

1. Leroy EM, Kumulungui B, Pourrut X, Rouquet $P$, Hassanin A, Yaba P, Delicat A, Paweska JT, Gonzalez JP, Swanepoel R: Fruit bats as reservoirs of Ebola virus. Nature 2005, 438(7068):575-576.

2. Peterson AT, Carroll DS, Mills JN, Johnson KM: Potential mammalian filovirus reservoirs. Emerg Infect Dis 2004, 10(12):2073-2081.

3. Barrette RW, Metwally SA, Rowland JM, Xu L, Zaki SR, Nichol ST, Rollin PE, Towner JS, Shieh WJ, Batten B, et al:: Discovery of swine as a host for the Reston ebolavirus. Science 2009, 325(5937):204-206.

4. Martina BE, Osterhaus AD: "Filoviruses": a real pandemic threat? EMBO Mol Med 2009, 1(1):10-18.

5. Walsh PD, Abernethy KA, Bermejo M, Beyers R, De Wachter P, Akou ME, Huijbregts B, Mambounga DI, Toham AK, Kilbourn AM, et al.: Catastrophic ape decline in western equatorial Africa. Nature 2003, 422(6932):611-614.

6. Kuzmin IV, Niezgoda M, Franka R, Agwanda B, Markotter W, Breiman RF, Shieh WJ, Zaki SR, Rupprecht CE: Marburg virus in fruit bat, Kenya. Emerg Infect Dis 2010, 16(2):352-354.

7. Strong JE, Wong G, Jones SE, Grolla A, Theriault S, Kobinger GP, Feldmann $\mathrm{H}$ : Stimulation of Ebola virus production from persistent infection through activation of the Ras/MAPK pathway. Proc Natl Acad Sci USA 2008, 105(46): 17982-17987.

8. Leroy EM, Epelboin A, Mondonge V, Pourrut X, Gonzalez JP, MuyembeTamfum JJ, Formenty P: Human Ebola outbreak resulting from direct exposure to fruit bats in Luebo, Democratic Republic of Congo, 2007. Vector Borne Zoonotic Dis 2009, 9(6):723-728.

9. Towner JS, Amman BR, Sealy TK, Carroll SA, Comer JA, Kemp A, Swanepoel $R$, Paddock CD, Balinandi S, Khristova ML, et al.: Isolation of genetically diverse Marburg viruses from Egyptian fruit bats. PLOS Pathog 2009, 5(7):e1000536.

10. Swanepoel R, Leman PA, Burt FJ, Zachariades NA, Braack LE, Ksiazek TG, Rollin PE, Zaki SR, Peters CJ: Experimental inoculation of plants and animals with Ebola virus. Emerg Infect Dis 1996, 2(4):321-325.

11. Morvan JM, Deubel V, Gounon P, Nakoune E, Barriere P, Murri S, Perpete $O$, Selekon B, Coudrier D, Gautier-Hion A, et al:: Identification of Ebola virus sequences present as RNA or DNA in organs of terrestrial small mammals of the Central African Republic. Microbes Infect 1999, 1(14):1193-1201.

12. Bente D, Gren J, Strong JE, Feldmann H: Disease modeling for Ebola and Marburg viruses. Dis Model Mech 2009, 2(1-2):12-17.

13. Holmes EC: The evolution and emergence of RNA viruses New York: Oxford University Press; 2009. 
14. Suzuki Y, Gojobori T: The origin and evolution of Ebola and Marburg viruses. Mol Biol Evol 1997, 14(8):800-806.

15. Sanchez A, Kiley MP, Klenk HD, Feldmann H: Sequence analysis of the Marburg virus nucleoprotein gene: comparison to Ebola virus and other non-segmented negative-strand RNA viruses. J Gen Virol 1992, 73(Pt 2):347-357.

16. Shi W, Huang Y, Sutton-Smith M, Tissot B, Panico M, Morris HR, Dell A, Haslam SM, Boyington J, Graham BS, et al: A filovirus-unique region of Ebola virus nucleoprotein confers aberrant migration and mediates its incorporation into virions. J Virol 2008, 82(13):6190-6199.

17. Horie M, Honda T, Suzuki Y, Kobayashi Y, Daito T, Oshida T, Ikuta K, Jern P, Gojobori T, Coffin JM, et al:: Endogenous non-retroviral RNA virus elements in mammalian genomes. Nature 2010, 463(7277):84-87.

18. Koonin EV: Taming of the shrewd: novel eukaryotic genes from RNA viruses. BMC Biol 2010, 8:2.

19. Emerman M, Malik HS: Paleovirology--modern consequences of ancient viruses. PLoS Biol 2010, 8(2):e1000301.

20. Taylor DJ, Bruenn J: The evolution of novel fungal genes from nonretroviral RNA viruses. BMC Biol 2009, 7:88.

21. Wiens JJ: Missing data and the design of phylogenetic analyses. $J$ Biomed Inform 2006, 39(1):34-42.

22. Geuking MB, Weber J, Dewannieux M, Gorelik E, Heidmann T, Hengartner $\mathrm{H}$, Zinkernagel RM, Hangartner L: Recombination of Retrotransposon and Exogenous RNA Virus Results in Nonretroviral cDNA Integration. Science 2009, 323(5912):393-396.

23. Watanabe $S$, Noda T, Kawaoka Y: Functional mapping of the nucleoprotein of Ebola virus. J Virol 2006, 80(8):3743-3751.

24. Zampieri CA, Sullivan NJ, Nabel GJ: Immunopathology of highly virulent pathogens: insights from Ebola virus. Nat Immunol 2007, 8(11):1159-1164.

25. Ebisuya M, Yamamoto T, Nakajima M, Nishida E: Ripples from neighbouring transcription. Nat Cell Biol 2008 in press.

26. Yang Z, Bielawski JP: Statistical methods for detecting molecular adaptation. Trends Ecol Evol 2000, 15(12):496-503.

27. Tamura K, Dudley J, Nei M, Kumar S: Molecular Evolutionary Genetics Analysis (MEGA) software version 4.0. Mol Biol Evol 2007 24(8):1596-1599.

28. Stern A, Doron-Faigenboim A, Erez E, Martz E, Bacharach E, Pupko T: Selecton 2007: advanced models for detecting positive and purifying selection using a Bayesian inference approach. Nucleic Acids Res 2007:W506-511

29. Khachane AN, Harrison PM: Assessing the genomic evidence for conserved transcribed pseudogenes under selection. Bmc Genomics 2009, 10:435.

30. Meredith RW, Westerman M, Springer MS: A phylogeny of Diprotodontia (Marsupialia) based on sequences for five nuclear genes. Mol Phylogenet Evol 2009, 51(3):554-571.

31. Adkins RM, Gelke EL, Rowe D, Honeycutt RL: Molecular phylogeny and divergence time estimates for major rodent groups: evidence from multiple genes. Mol Biol Evol 2001, 18(5):777-791.

32. Springer MS, Murphy WJ, Eizirik E, O'Brien SJ: Placental mamma diversification and the Cretaceous-Tertiary boundary. Proc Natl Acad Sci USA 2003, 100(3):1056-1061

33. Dereeper A, Audic S, Claverie JM, Blanc G: BLAST-EXPLORER helps you building datasets for phylogenetic analysis. BMCEVol Biol 2010, 10:8

34. Katoh K, Misawa K, Kuma K, Miyata T: MAFFT: a novel method for rapid multiple sequence alignment based on fast Fourier transform. Nucleic Acids Res 2002, 30(14):3059-3066.

35. Talavera G, Castresana J: Improvement of phylogenies after removing divergent and ambiguously aligned blocks from protein sequence alignments. Syst Biol 2007, 56(4):564-577.

36. Gouy M, Guindon S, Gascuel O: SeaView version: A multiplatform graphical user interface for sequence alignment and phylogenetic tree building. Mol Biol Evol 2010, 27(2):221-224

37. Stamatakis A: RAxML-VI-HPC: maximum likelihood-based phylogenetic analyses with thousands of taxa and mixed models. Bioinformatics 2006, 22(21):2688-2690

38. Ronquist F, Huelsenbeck JP: MrBayes 3: Bayesian phylogenetic inference under mixed models. Bioinformatics 2003, 19(12):1572-1574.

39. Abascal F, Zardoya R, Posada D: ProtTest: selection of best-fit models of protein evolution. Bioinformatics 2005, 21(9):2104-2105.
40. Stamatakis A, Hoover P, Rougemont J: A rapid bootstrap algorithm for the RAxML Web servers. Syst Biol 2008, 57(5):758-771.

41. Suyama M, Torrents D, Bork P: PAL2NAL: robust conversion of protein sequence alignments into the corresponding codon alignments. Nucleic Acids Res 2006:W609-612.

42. Sinha AU, Meller J: Cinteny: flexible analysis and visualization of synteny and genome rearrangements in multiple organisms. $B m C$ Bioinformatics 2007, 8:82.

43. Deluca TF, Wu IH, Pu J, Monaghan T, Peshkin L, Singh S, Wall DP: Roundup: a multi-genome repository of orthologs and evolutionary distances. Bioinformatics 2006, 22(16):2044-2046.

44. Blanga-Kanfi S, Miranda H, Penn O, Pupko T, DeBry RW, Huchon D: Rodent phylogeny revised: analysis of six nuclear genes from all major rodent clades. BMC Evol Biol 2009, 9:71

45. Murphy WJ, Pringle TH, Crider TA, Springer MS, Miller W: Using genomic data to unravel the root of the placental mammal phylogeny. Genome Res 2007, 17(4):413-421.

doi: 10.1186/1471-2148-10-193

Cite this article as: Taylor et al., Filoviruses are ancient and integrated into mammalian genomes BMC Evolutionary Biology 2010, 10:193

\section{Submit your next manuscript to BioMed Central and take full advantage of:}

- Convenient online submission

- Thorough peer review

- No space constraints or color figure charges

- Immediate publication on acceptance

- Inclusion in PubMed, CAS, Scopus and Google Scholar

- Research which is freely available for redistribution
C Biomed Central 